\title{
Gerador trifásico de baixo custo para o ensino de física
}

\author{
Low-cost three-phase generator for physics teaching
}

\author{
Giovani Teixeira de Azevedo, Carlos José Todero Peixoto, Fabiano Fernandes Bargos, Carlos \\ Renato Menegatti*
}

Escola de Engenharia de Lorena, Universidade de São Paulo, Lorena, SP, Brasil

\begin{abstract}
Recebido em 06 de Dezembro, 2016. Revisado em 03 de janeiro, 2017. Aceito em 04 de Janeiro, 2017
\end{abstract}
\begin{abstract}
Neste artigo apresentamos um aparato experimental, construído com materiais de baixo custo, para o estudo de geradores trifásicos e circuitos de corrente alternada. Com este aparato é possível realizar diversos experimentos em regime c.a., os quais serão propostos no decorrer do artigo. Além disso, o aparato pode ser configurado para gerar diversas formas de onda com tensões de pico da ordem de 1 V. Assim, o estudante será capaz de observar efeitos de distorção harmônica do sinal do gerador e a sua influencia na potência média máxima transferida. O aparato também pode ser usado como uma ferramenta demonstrativa auxiliando na compreensão fenomenológica da lei de indução de Faraday e pode ser usado tanto em laboratórios de ensino quanto em salas de aula.
\end{abstract}

Palavras-chave: gerador trifásico, circuitos em c.a., distorção harmônica, experimento demonstrativo.

In this paper we present an experimental set up, built with low cost materials, for studying three-phase generators and alternating current circuits. The setup allows several experiments in c.a. regime to be carried out, which will be proposed throughout the article. In addition, the setup can be arranged to generate several waveforms with peak voltages in the order of $1 \mathrm{~V}$. The student can observe harmonic distortion effects of the generator signal and its influence on the maximum average power transferred. The setup can also be used as a demonstrative tool for assisting in the phenomenological understanding of Faraday's induction law and can be used in either classrooms or laboratories.

Keywords: three-phase generator, a.c. circuits, harmonic distortion, demonstrative experiment.

\section{Introdução}

A experimentação é um componente essencial no ensino de ciências e engenharia. Diversos fenômenos e conceitos podem ser mais facilmente assimilados quando o aluno participa com regularidade em atividades experimentais [1]. Enquanto a experiência em sala de aula é importante para compreensão dos fundamentos teóricos, a aprendizagem aplicada sobre o equipamento é essencial para que o estudante tenha experiência com problemas reais.

Existem várias condições necessárias para um bom aproveitamento em atividades de experimentação. A Associação Americana de Professores de Física elaborou uma lista de objetivos de aprendizagem para as atividades laboratoriais na qual indica as condições necessárias. Dentre as condições aponta-

*Endereço de correspondência: renatomenegatti@usp.br das está o envolvimento dos alunos nestas atividades [2]. Com as experiências "hands-on"os alunos também podem manusear e manipular, o que aumenta a motivação, a concentração, a autonomia na resolução de problemas e o gosto pela ciência $[3,4]$.

A eletricidade é um ramo da física essencialmente experimental no qual as atividades práticas tem papel de destaque no desenvolvimento de diversas competências e habilidades. Nesse sentido, os laboratórios de ensino de eletricidade são fundamentais para o desenvolvimento do estudante, principalmente para os estudantes das áreas tecnológicas, como a engenharia. No entanto, a construção desses laboratórios é cara e demorada, além disso, eles requerem manutenção permanente, o que exige o constante trabalho de técnicos treinados. A construção de equipamentos versáteis, que sejam de fácil adaptação para diferentes configurações e experi- 
mentos, é uma das alternativas viáveis para reduzir o custo dos laboratórios.

Nesse trabalho, apresentamos um gerador de corrente alternada trifásico de baixo custo que permite a elaboração de diversos experimentos relacionados às disciplinas de eletricidade básica e aplicada. Por ser de baixo custo, e de fácil construção e adaptação, pode ser empregado tanto no ensino médio e técnico quanto em cursos tecnológicos e de graduação. O aparato pode ser usado em laboratórios de ensino e em atividades demonstrativas em sala de aula. Além dos detalhes da construção do gerador, são apresentadas propostas de atividades experimentais elaboradas com o objetivo de exemplificar o emprego do equipamento em assuntos diferentes dentro do tema corrente alternada.

\section{Considerações Teóricas}

O princípio de funcionamento de diversos dispositivos e sistemas eletro - eletrônicos de uso diário baseia-se na lei da indução descoberta há quase dois séculos. Michael Faraday verificou que campos magnéticos variáveis induziam uma diferença de potencial alternada entre os extremos de uma espira condutora. Nas últimas duas décadas do século XIX houve uma grande disputa entre George Westinghouse e Thomas Edison [5, 6], nesta disputa George defendia a utilização de corrente alternada para distribuição de eletricidade em contrapartida com Thomas Edison que defendia a utilização de corrente contínua. Esta disputa ficou conhecida historicamente como Guerra das Correntes. Em 1887, quando Tesla introduziu o sistema de geradores e transformadores a corrente alternada, tornou-se claro, devido as suas grandes vantagens, que esse tipo de corrente era o futuro para o sistema de distribuição de energia elétrica, embora o sistema contínuo fosse utilizado nos centros das áreas metropolitanas por décadas subsequentes.

A corrente alternada pode ser entendida pela lei da indução de Faraday [7], a qual afirma que a variação do fluxo de campo magnético $(\phi)$ através de uma bobina com $N$ espiras induz uma diferença de potencial $(v)$ entre suas extremidades, podendo ser descrita pela equação (11):

$$
v(t)=-N \frac{d \phi(t)}{d t}
$$

É de nosso conhecimento que a tensão gerada e distribuída em nossa sociedade é alternada com forma senoidal, $v(t)=v_{0} \operatorname{sen}(\omega t+\theta)$. Assim, para um gerador produzir esta tensão é necessário que $\phi(t)$ tenha um comportamento de uma função cosseno. A amplitude da tensão gerada $\left(v_{0}\right)$ será proporcional ao campo magnético dos magnetos, a área da seção transversal e o número de voltas das bobinas.

Um sistema CA monofásico é formado por um gerador conectado por meio de um par de fios a uma carga. A tensão produzida é caracterizada por uma amplitude $v_{0}$ e uma fase $\theta$. A potência média que um sistema monofásico produz pode ser calculada conforme:

$$
P_{\text {med }}=\frac{1}{T} \int_{0}^{T} v(t) i(t) d t=\frac{1}{2} v_{0} i_{0} \cos \left(\theta_{v}-\theta_{i}\right)
$$

Em que $\mathrm{v}_{0}$ e $\mathrm{i}_{0}$ são as respectivas amplitudes da tensão e corrente e $\theta_{\mathrm{v}}-\theta_{\mathrm{i}}$ é a diferença de fase.

Em sistemas trifásicos [8] um gerador é formado por três bobinas de mesma amplitude e frequência, porém defasadas entre si por $120^{\circ}$. Dessa forma são produzidas três tensões: $v_{A}, v_{B}, v_{C}$ :

$$
\begin{aligned}
& v_{A}(t)=\cos (\omega t) ; \\
& v_{B}(t)=\cos \left(\omega t+120^{\circ}\right) ; \\
& v_{C}(t)=\cos \left(\omega t+240^{\circ}\right)
\end{aligned}
$$

O princípio de funcionamento de sistemas trífasicos é o mesmo que o monofásico. Sua principal diferença está no arranjo de suas bobinas onde são posicionadas 3 bobinas ao redor de um rotor magnético.

\section{Aparato Experimental}

O aparato experimental do gerador foi projetado baseado em componentes de baixo custo e de maneira que pudéssemos obter um kit didático compacto que produzisse tensões de pico da ordem de 1 Volt.

Primeiramente foi projetado um rotor em alumínio com diâmetro de $60 \mathrm{~mm}$ (Figuras 1a, 1d e 1e) com possibilidade de alocação de até 8 pequenos ímãs. Os ímãs eram de neodímio em formato cilíndrico (diâmetro de $10 \mathrm{~mm}$ e $4 \mathrm{~mm}$ de espessura) e a intensidade do campo magnético de cada ímã era de aproximadamente 500 Gauss a $5 \mathrm{~mm}$ de sua face (dados fornecidos pelo fabricante - KOIMÃS). Ao redor do rotor foram feitos 8 rebaixos para que os ímãs pudessem ser fixados. Em cada rebaixo havia um parafuso de ferro de modo que os ímãs se fixavam magneticamente. Com este arranjo a troca e reposicionamento dos ímãs podiam ser feitas de maneira muito prática possibilitando a criação de 


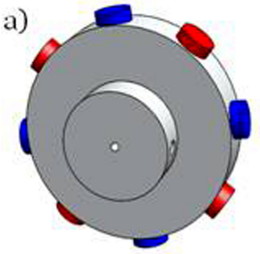

b)
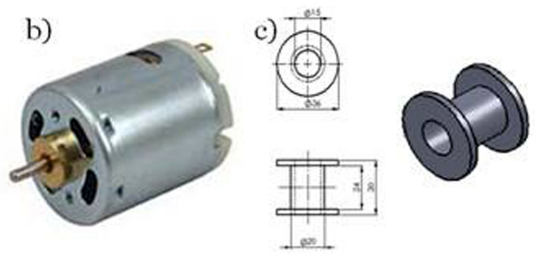

d)

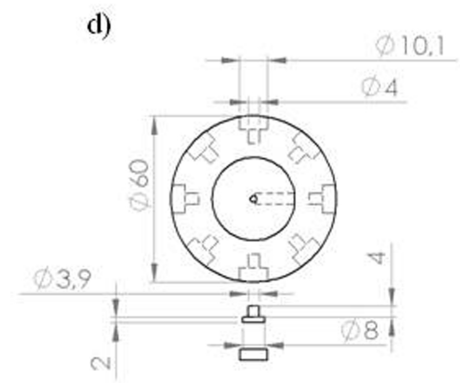

e)

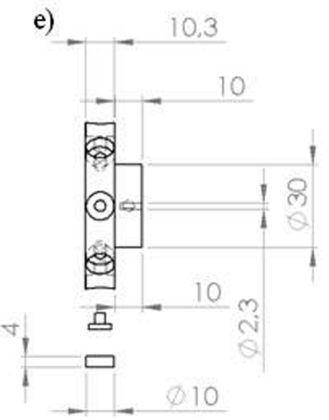

Figura 1: a) Rotor de alumínio com possibilidade de alocação de 8 ímãs; b) Motor c.c. de 12 V; c) Suporte das bobinas; d) e e) Diagrama do corpo do rotor com parafuso de material ferromagnético e ímã (medidas em $\mathrm{mm})$.

diversas configurações de linhas de campo magnético ao redor do rotor. Por fim, esta peça de alumínio era acoplada a um pequeno motor c.c. de 12 Volts com rotações de até $12000 \mathrm{rpm}$ (Figura 1b).

$\mathrm{Na}$ Figura 1c temos o suporte das bobinas. O suporte foi usinado em nylon em formato cilíndrico com as seguintes dimensões: diâmetro de $15 \mathrm{~mm}$ e comprimento de $30 \mathrm{~mm}$. Para os enrolamentos foi utilizado fio de cobre esmaltado AWG 24, cada bobina possuía 1000 voltas. Os valores destas grandezas foram estimados considerando o valor de campo de cada ímã, tensão de pico da ordem $1 \mathrm{~V}$ e frequência da ordem de $100 \mathrm{~Hz}$.

Todos estes componentes foram montados em uma placa de acrílico compondo o kit experimental da Figura 2. O motor c.c. foi fixado embaixo da placa por uma camisa cilíndrica feita em nylon e as

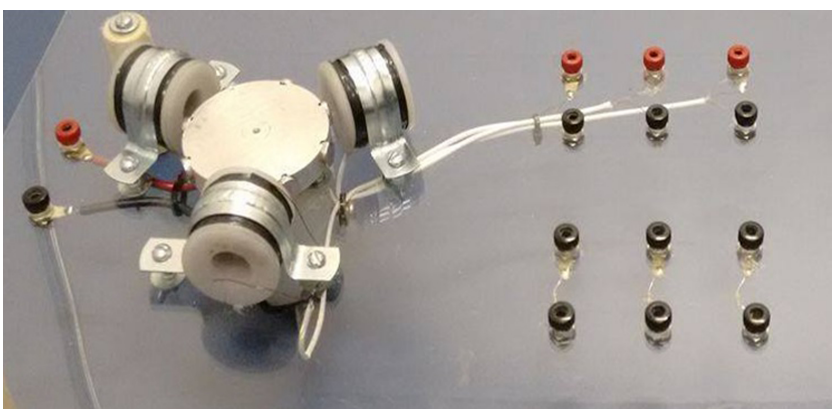

Figura 2: Kit experimental. bobinas foram fixadas com abraçadeiras de alumínio. Para as conexões elétricas foram utilizados bornes. Nos bornes do lado esquerdo da placa era conectado uma fonte c.c. variável para alimentação do motor elétrico. Os três pares de bornes do lado direito superior foram conectados as tensões geradas por cada bobina e os três pares do lado direito inferior foram conectados cargas resistivas. É importante ressaltar que todo o conjunto teve orçamento de aproximadamente $\mathrm{R} \$ 100,00$.

\section{Atividades propostas em aula}

Nesta seção apresentaremos algumas propostas de utilização do gerador trifásico. Basicamente propomos 4 estudos: a) Estudo das formas de onda induzidas pelo gerador; b) Relação tensão induzida $\mathrm{x}$ frequência do rotor; c) Medida da potência média máxima produzida no gerador em função da carga e em função da forma de onda gerada; d) Observação simultânea das fases geradas e a verificação da relação das tensões de fase com tensões de linha.

\subsection{Estudo das formas de onda induzidas pelo gerador.}

O primeiro experimento proposto é baseado na observação experimental das formas de onda produzida por apenas uma bobina em função da configuração de ímãs do rotor. Como descrito na seção anterior, os ímãs se acoplam nos rebaixos magneticamente deixando o experimento flexível. Assim foram exploradas 5 configurações de ímãs como mostrado na Figura 3.

Utilizando um osciloscópio foi medido o sinal induzido em uma das bobinas para cada arranjo. Para este experimento forma induzidas tensões com frequências de aproximadamente $80 \mathrm{~Hz}$ (Figura 4).

As cinco formas de onda obtidas na Figura 4 são devidas as respectivas configurações de ímãs mencionadas na Figura 3. Com este resultado o estudante pode ter uma experiência visual da relação da distribuição espacial de campo magnético com a tensão induzida. Cada forma de onda, apesar de não intuitivas, podem ser explicadas utilizando-se diretamente a lei de indução de Faraday. Neste ponto o estudante vai perceber que quanto mais homogênea a distribuição de campo, mais próximo de uma senóide o sinal se comportará. Com estes resultados podese até mesmo exigir que o aluno faça uma decomposição espectral dos sinais aplicando transformada 
discreta de Fourier [9] para determinar a amplitude dos harmônicos que compõe a onda. Para esta tarefa podem ser utilizados programas como o Origin ou Mathematica que calculam a transformada rápida de Fourier (FFT - Fast Fourier Transformer) ou mesmo utilizando funções matemáticas que alguns modelos de osciloscópios digitais possuem.

\subsection{Relação tensão induzida $\mathrm{x}$ frequência do rotor.}

Deve ser de conhecimento do estudante que, pela lei de indução de Faraday, quanto maior a taxa de variação do campo magnético em uma bobina maior será a tensão induzida pelo mesmo. Assim uma forma de ilustrar este fenômeno é variar a frequência do rotor e medir a tensão de pico induzido. $\mathrm{Na}$ Figura 5 temos uma medida da tensão de pico e frequência da onda gerada em função da tensão aplicada ao motor c.c. A forma de onda utilizada foi a forma senoidal, configuração de 8 ímãs alternados (Figura 4c).

Com este resultado percebe-se claramente uma relação linear entre a tensão do motor c.c. com a tensão de pico e frequência do sinal induzido. Este comportamento linear sugere que o gerador também possa ser utilizado como um gerador de frequência. A frequência máxima obtida neste aparato foi de $800 \mathrm{~Hz}$. Assim, conhecendo-se a tensão do motor c.c. pode-se determinar a frequência e amplitude da onda por interpolação.

a)

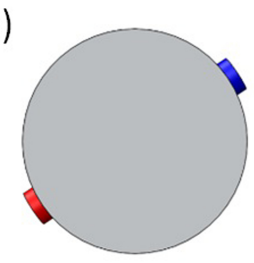

b)
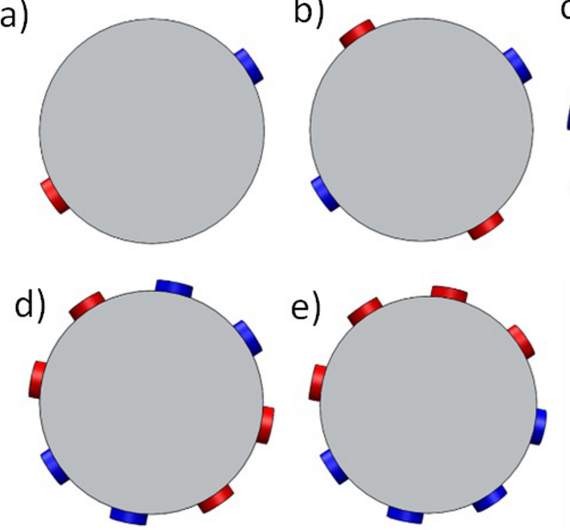

Figura 3: Configurações dos ímãs no rotor: (a) 2 ímãs; (b) 4 ímãs; (c) 8 ímãs; (d) 8 ímãs alternados 2 norte 2 sul; (e) 8 ímãs alternados 4 norte 4 sul. As cores azul e vermelha representam a polaridade da face superior de cada ímã.

\subsection{Medida da potência média máxima produzida no gerador em função da carga e em função da forma de onda}

Para a determinação da máxima potência produzida por uma das bobinas podemos conectar sua tensão em uma carga resistiva variável $\left(\mathrm{R}_{\mathrm{c}}\right)$. Medindo-se a tensão de pico na carga com o osciloscópio ou mesmo a tensão RMS com um multímetro, pode-se calcular a potência média dissipada como:

$$
P_{\text {med }}=\frac{V_{p}^{2}}{2 R_{c}}=\frac{V_{r m s}^{2}}{R_{c}}
$$

Ao variar a carga do circuito de 2 até $300 \Omega$ podese observar o comportamento da potência como mostrado pelos pontos experimentais no gráfico da Figura 6. A potência média deste circuito pode ser representada matematicamente em função da resistência da carga $R_{c}$ como:

$$
P_{\text {med }}\left(R_{c}\right)=\frac{R_{c}}{\left(R_{c}+R_{\text {int }}\right)^{2}} V_{r m s}^{2}
$$

Em que $R_{\text {int }}$ é a resistência interna de uma bobina podendo ser determinada de três formas: a) medindo-se diretamente a resistência da bobina com um multímetro; b) ajustando os pontos experimentais pela equação 5 ; c) sabendo que a máxima transferência de potência de um circuito acontece quando $\mathrm{R}_{\mathrm{c}}=\mathrm{R}_{\mathrm{int}}, \mathrm{R}_{\text {int }}$ pode ser determinada graficamente. No caso do ajuste dos pontos experimentais da figura 6 pela equação 5 foram obtidos $\mathrm{V}_{\mathrm{rms}}=$ $0,64 \mathrm{~V}$ e $\mathrm{R}_{\text {int }}=15 \Omega$ com desvio padrão de $0,5 \%$. Medindo-se a resistência da bobina com o ohmímetro foi obtido $\mathrm{R}_{\text {int }}=15,1 \Omega$. Isso mostra que este experimento gera resultados consistentes.

Neste experimento o estudante também poderá fazer um estudo da potência média gerada em função da resistência da carga para cada forma de onda produzida (curvas coloridas na Figura 6b). Nota-se que a configuração de ímãs chamada de 4 norte 4 sul produz a menor potência máxima enquanto que a forma de onda senoidal produz a máxima (nesta medida ajustamos todas as formas de onda para uma mesma tensão de pico de aproximadamente 0,9 V). Com esta medida, fica evidente que quanto mais distorcida a onda, ou seja, quanto mais harmônicos ela possuir pior será a eficiência do gerador. Geralmente essa ineficiência está associada a taxa de distorção harmônico (THD) [10, 11] de uma onda 
que pode ser calculada como:

$$
T H D=\frac{\sqrt{\sum_{2}^{n} V_{i}^{2}}}{V_{1}} \times 100
$$

Nesta equação $V_{1}$ é amplitude do modo fundamental e $V_{i}$ a amplitude dos harmônicos do sinal. Com base nesta definição, o aluno poderá qualificar a tensão do gerador atribuindo uma taxa de distorção harmônica para cada configuração de magnetos. Este tópico é muito interessante e pouco é abordado em cursos de física e eletricidade.

\subsection{Observação simultânea das fases geradas e a verificação da relação das tensões de fase com tensões de linha.}

Nesta última proposta, o estudante primeiramente poderá visualizar as três ondas geradas simultaneamente utilizando um osciloscópio com 4 canais ou com 2 canais. A Figura 7 representa uma medida a)

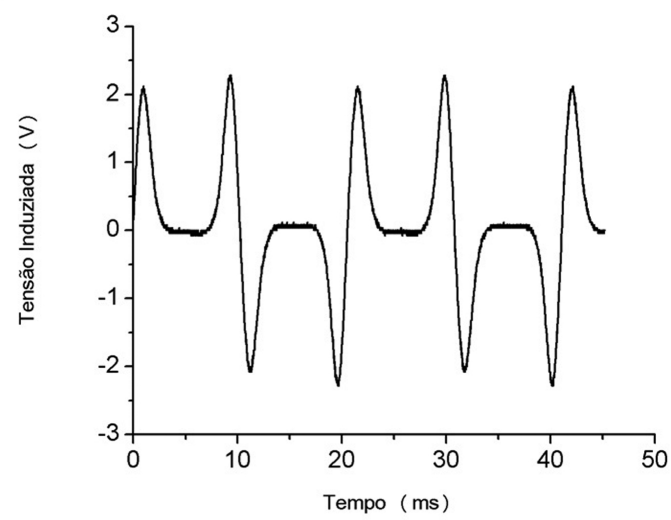

c)

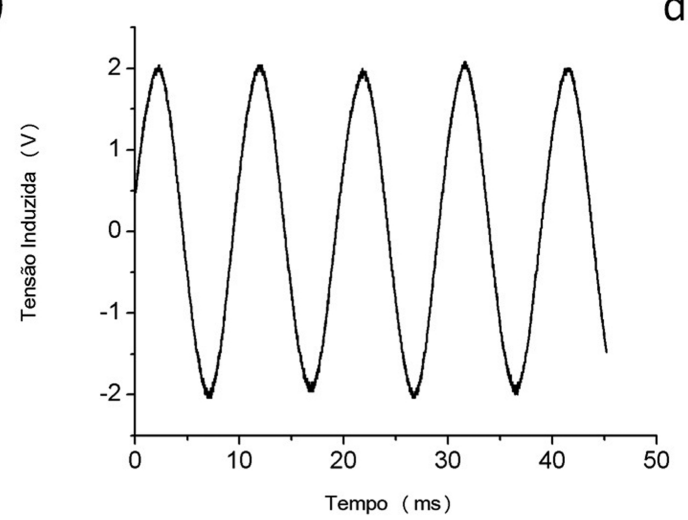

e)

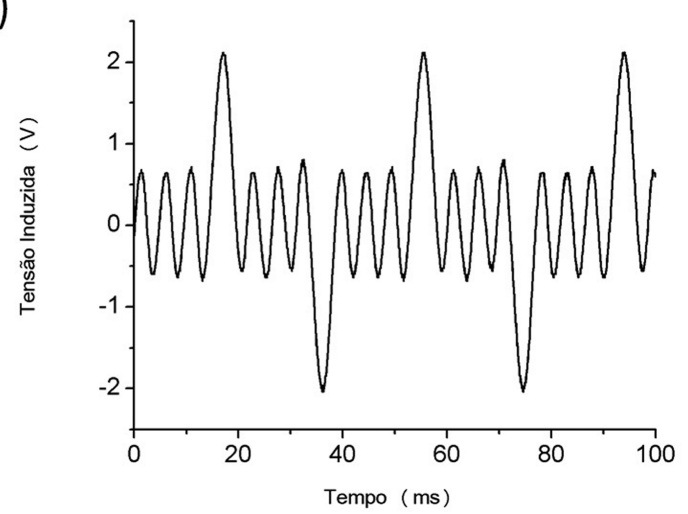

b)

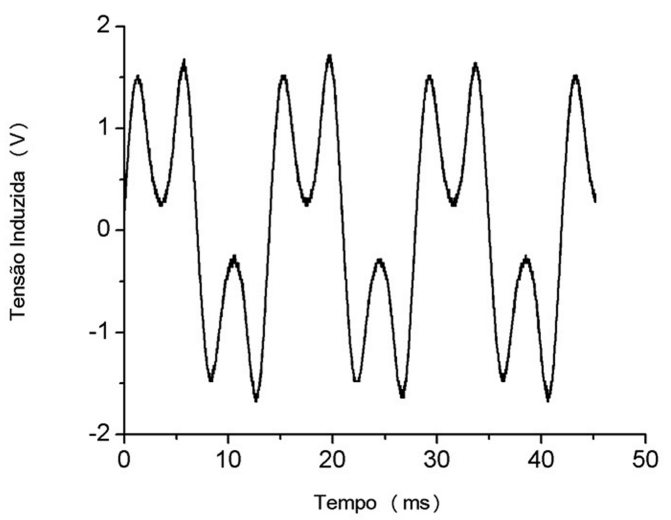

d)

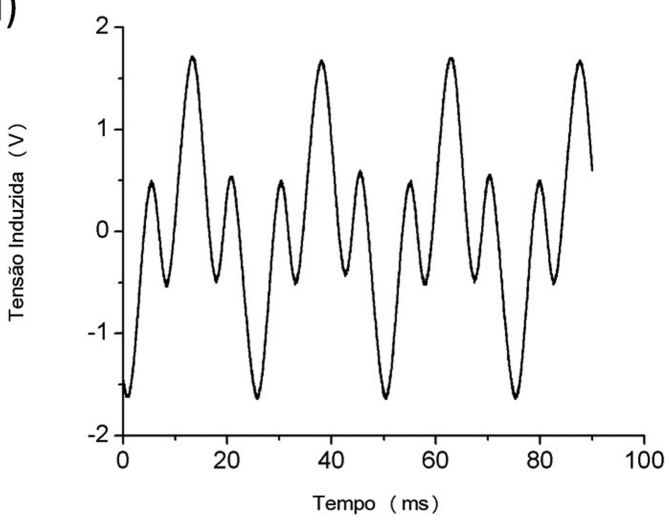

Figura 4: Tensão gerada em função da configuração de ímãs: (a) 2 ímãs; (b) 4 ímãs; (c) 8 ímãs; (d) 8 ímãs alternados 2 norte 2 sul; (e) 8 ímãs alternados 4 norte 4 sul. 
simultânea das três bobinas. O estudante pode determinar a diferença de fase entre as ondas utilizando a expressão $\Delta \varphi=360 \Delta \mathrm{t} / \mathrm{T}$, em que $\Delta \mathrm{t}$ é o intervalo de tempo entre o pico de duas ondas e $\mathrm{T}$ o período da onda.

Diversas configurações de tensão de fase e carga podem ser utilizadas neste experimento, uma delas pode ser obtida pela conexão estrela-estrela representada na Figura 8. Nesta configuração pode-se medir e comparar a amplitude das tensões de fase $\mathrm{V}_{\mathrm{f}}$ com as tensões de linha $\mathrm{V}_{\mathrm{L}}$ que possuem uma relação dada por:

$$
V_{L}=\sqrt{3} V_{f}
$$

Outra característica que fazem dos sistemas trifásicos vantajosos com relação aos monofásicos é que sua potência instantânea total é constante no tempo,

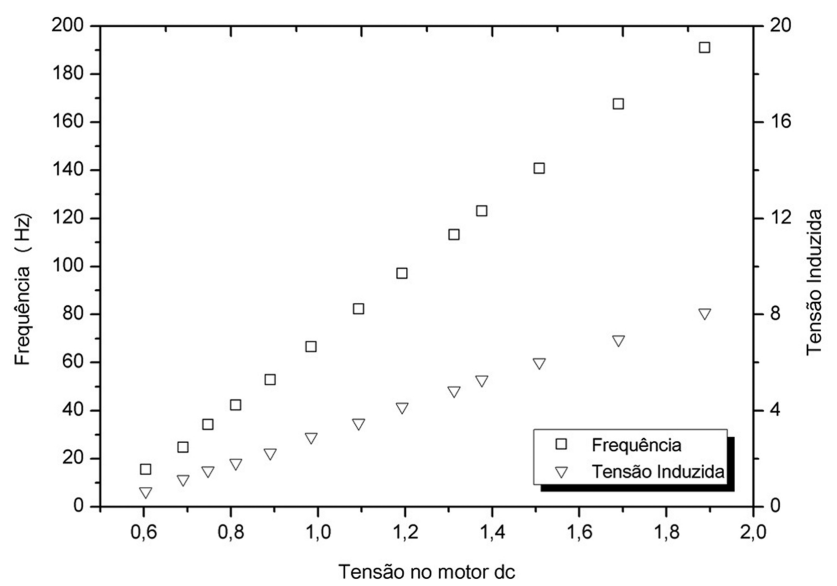

Figura 5: Tensão de pico e frequência da onda induzida em função da tensão do motor c.c. A forma de onda utilizada neste experimento foi a forma senoidal.

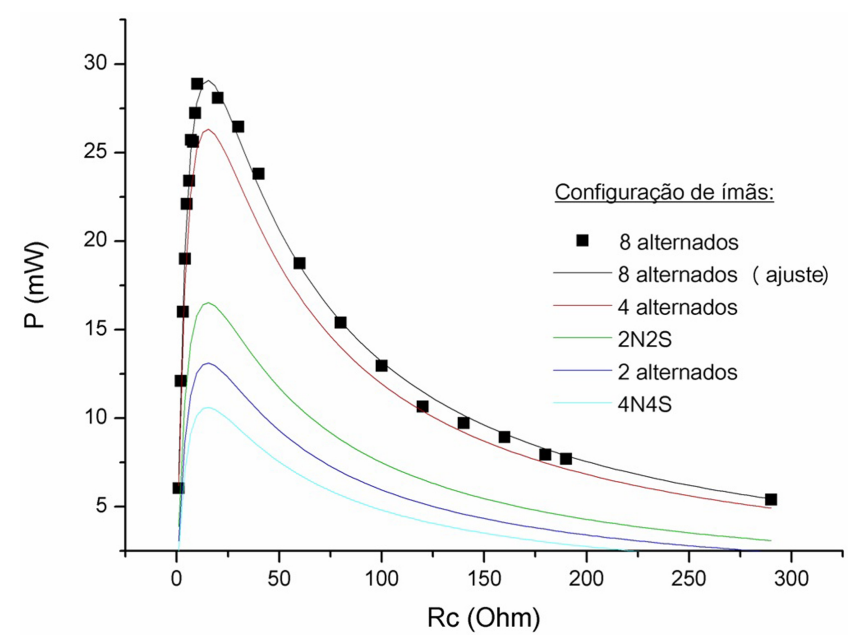

Figura 6: Potência média produzida em função da resistência da carga e da forma de onda induzida.

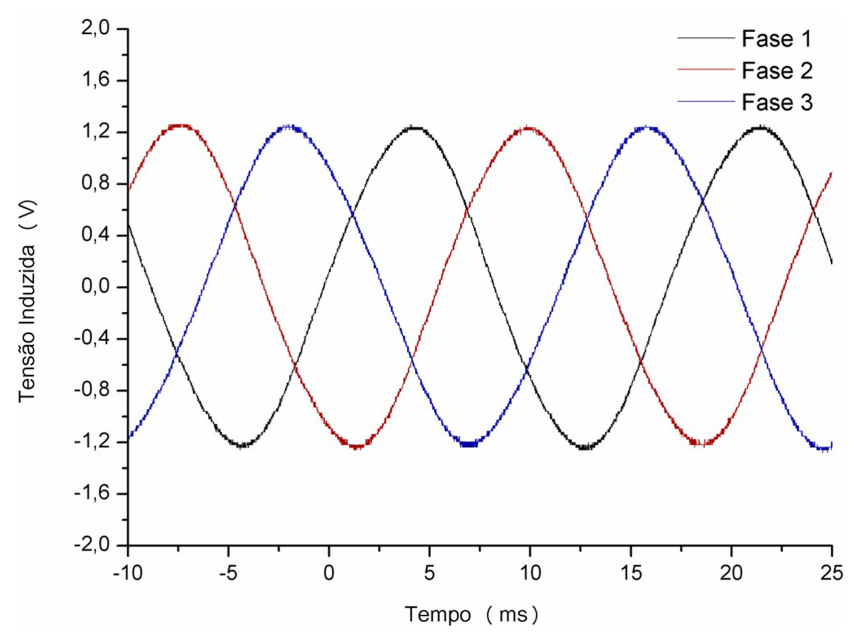

Figura 7: Medida simultânea das tensões geradas pelas 3 bobinas.

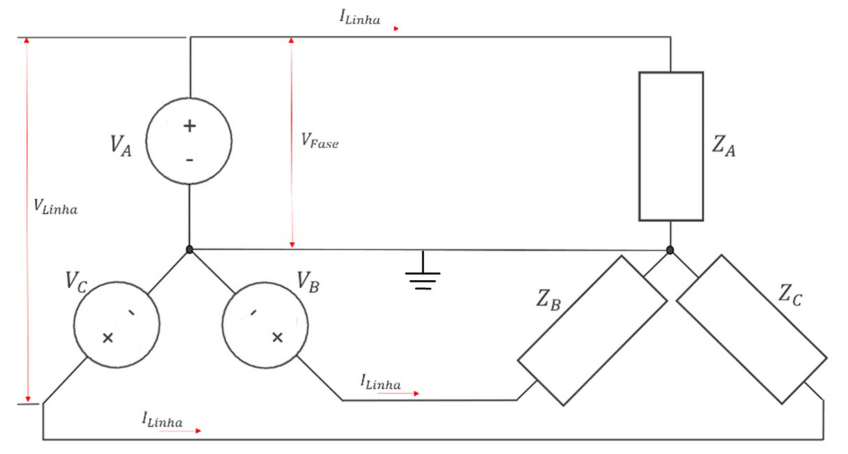

Figura 8: Medida simultânea das tensões geradas pelas 3 bobinas.

pode-se demonstrar que a potência instantânea total é dada por:

$$
p(t)=p_{a}(t)+p_{b}(t)+p_{c}(t)=3 V_{f} I_{f} \cos \theta
$$

Em que $\mathrm{p}_{\mathrm{i}}(\mathrm{t})$ são as potências instantânea de cada fase, $V_{\mathrm{f}}$ e $\mathrm{I}_{\mathrm{f}}$ são os respectivos valores da tensão e corrente de fase dados em RMS e $\theta$ a diferença de fase entre ambos. No caso de cargas resistivas podemos considerar que a diferença de fase $\theta$ é nula, assim:

$$
p(t)=\frac{1}{R}\left(v_{a}^{2}(t)+v_{b}^{2}(t)+v_{c}^{2}(t)\right)=3 \frac{V_{f}^{2}}{R}
$$

Desse modo, um gráfico da soma dos quadrados das tensões medidas em função do tempo deve se aproximar de uma constante no caso de três bobinas idênticas defasadas exatamente a $120^{\circ}$.

\section{Considerações Finais}

Neste trabalho foi apresentado uma alternativa para o estudo de geradores trifásicos. O Kit experimental 
construído com materiais de baixo custo, aproximadamente $\mathrm{R} \$ 100,00$, demostrou ser robusto, versátil e com grande potencial de ser implementado em cursos experimentais de física e engenharia. O aparato poderá ser empregado de maneira demonstrativa ou analítica auxiliando na compreensão fenomenológica da lei de indução de Faraday e na geração de tensão alternada senoidal. A possibilidade de alteração da configuração de magnetos torna possível também a geração e estudo de ondas distorcidas. Através da terceira proposta experimental, fica evidente o efeito das distorções harmônicas na potência média de um gerador, mostrando ao estudante a importância de se produzir tensões senoidais.

Podemos ressaltar que este kit experimental não fica limitado apenas aos experimentos propostos aqui. No segundo experimento, notamos uma relação linear entre frequência e tensão do motor c.c. Podemos elevar a rotação do rotor de modo a obtermos frequências de no máximo $800 \mathrm{~Hz}$. Desse modo, pode-se criar diversas possibilidades de utilização em experimentos em regime c.a. como por exemplo: o estudo da resposta em frequência de um circuito RC; Estudo de potência em c.a.: potência aparente, fator de potência, correção de fator de potência, etc.

Finalizando, as propostas de atividades experimentais e demonstração, utilizando o kit experimental, podem estimular a participação e o envolvimento dos alunos contribuindo para o ensino e a aprendizagem de geradores e tensão alternada.

\section{Referências}

[1] Avi Ornstein, Journal of Science Education and Technology 15, 285 (2006).

[2] American Association of Physics Teachers, Role of Labs in High School Physics, disponível em http://www.aapt.org/resources/policy/ Role0fLabs.cfm, acessado em 1/12/2016.

[3] L. Haury and P. Rillero, Perspectives of Hands-On Science Teaching, North Central Regional Educational Laboratory's, disponível em http://eric.ed. gov/?id=ED372926, acessado em 1/12/2016.

[4] Filipa Oliveira e José António Paixao, Rev. Bras. Ensino Fís. 39, e1402 (2017).

[5] AC Power History. Edison Tech Center, disponível em http://www.edisontechcenter.org/ AC-PowerHistory.html, acessado em 3/1/2017.

[6] Jill Jonnes, Empires Of Light: Edison, Tesla, Westinghouse, And The Race To Electrify The World (Random House, New York, 2004).
[7] H.M. Nussenzveig, Eletromagnetismo, Curso de Física Básica, vol. 3 (Editora Edgard Blücher LTDA, São Paulo, 1997).

[8] M.N.O. Sadiku, Fundamentals of Eletric Circuits (Mcgraw Hill, Nova Iorque, 2013), $5^{\mathrm{a}}$ ed.

[9] C. Scherer, Métodos Computacionais da Fésica (Livrariada da Física, São Paulo, 2010), 299 p.

[10] André Luis Zago de Grandi, Metodologia para Avaliação de Distorção Harmônica de Corrente em Transformadores de Distribuição. Dissertação de Mestrado em Sistemas de Potência, Escola Politécnica, Universidade de São Paulo, São Paulo, 2012.

[11] Douglas Ângelo Teixeira, Análise das Distorções Harmônicas - Estudo de Caso de um Sistema Industrial. Dissertação de Mestrado, Universidade Federal de Minas Gerais, Belo Horizonte, 2009. 